\title{
36. UNA NUEVA COMBINACIÓN EN EL GÉNERO PODOSPERMUM DC. (ASTERACEAE)
}

\author{
Consuelo DÍAZ DE LA GUARDIA* y Gabriel BLANCA
}

Recibido el 30 de septiembre de 2015, aceptado para su publicación el 15 de octubre de 2015

A new combination in the genus Podospermum DC. (Asteraceae)

Palabras clave. Podospermum, Scorzonera, taxonomía, Lactuceae, Asteraceae, Compositae

Key words. Podospermum, Scorzonera, taxonomy, Lactuceae, Asteraceae, Compositae

El género Podospermum DC. incluye alrededor de 10 especies distribuidas por Eurasia y N de África, caracterizadas por tener aquenios con un podocarpo en la base. Aunque a menudo se ha considerado como subgénero de Scorzonera L., ya que el podocarpo está esbozado en algunas especies de este género (Díaz de la Guardia \& Blanca, 1987), estudios de filogenia molecular sugieren su consideración como género independiente, al tiempo que indican que el género Scorzonera en sentido estricto es polifilético (Mavrodiev et al., 2004, 2012; Owen et al., 2006).

En la Flora iberica solo habita una especie, Podospermum laciniatum (L.) DC., cuya enorme variabilidad fue descrita por Díaz de la Guardia \& Blanca (1987). Aunque los caracteres florales son muy constantes, el porte, la cantidad de indumento y, sobre todo, la morfología de las hojas son muy variables, lo que ha originado la propuesta de diversos táxones infraespecíficos, que los mencionados autores sintetizaron a tres con rango varietal, ya que todas las variantes observadas solapan geográficamente y existen numerosas formas de tránsito: var. laciniatum, de hojas pinnatipartidas o pinnatisectas, con segmentos lineares, linear-lanceolados a oval- lanceolados, abundante por todo el territorio, aunque son los ejemplares de segmentos lineares los que están mejor representados en el centro peninsular; var. calcitrapifolia (Vahl) Moris, de hojas igualmente pinnatipartidas o pinnatisectas, pero con segmentos de obovadooblongos a orbiculares, de margen undulado y ápice obtuso, que es también frecuente en la mayor parte del territorio, sobre todo en la mitad sur de la Península; y la var. subulatum (Lam.) DC., de hojas enteras, lineares o linearlanceoladas, mucho más escasa, propia de saladares, yesares o de suelos muy pobres.

Para la var. calcitrapifolia existe una denominación prioritaria con el rango varietal, Scorzonera calcitrapifolia var. decumbens Guss., por lo que se propone la siguiente combinación:

Podospermum laciniatum var. decumbens (Guss.) C. Díaz \& Blanca, comb. nova $\equiv$ Scorzonera calcitrapifolia var. decumbens Guss., P1. Rar.: 323 (1826), basión.

$\equiv S$. decumbens (Guss.) Guss., F1. Sicul. Syn. 2: 386 (1843)

$\equiv$ P. decumbens (Guss.) Gren. \& Godr.,

Este trabajo ha sido desarrollado en el contexto de la obra "Flora iberica" y financiado por el proyecto CGL2012-32914, de la Dirección General de Investigación Científica y Técnica del Ministerio de Economía y Competitividad, cofinanciado con fondos FEDER. 
Fl. France 2: 310 (1850)

$\equiv$ S. laciniata var. decumbens (Guss.) Fiori, Nuov. Fl. Italia 2: 808 (1928)

$\equiv S$. laciniata subsp. decumbens (Guss.)

Greuter in Willdenowia 33: 237 (2003)

$\equiv$ P. laciniatum subsp. decumbens (Guss.)

Gemeinholzer \& Greuter in Willdenowia 36(2): 715 (2006)

$=$ S. resedifolia L., Sp. Pl. 2: 1198 (1753)

$\equiv$ P. resedifolium (L.) DC. in Lam. \& DC., Fl. Franç. ed. 3, 4(1): 61 (1805)

$\equiv$ P. decumbens var. resedifolium (L.) Gren.

\& Godr., Fl. France 2: 310 (1851)

$=$ S. plurifida Lam., Fl. Franç. ed. 1, 2: 83 (1779)

= S. calcitrapifolia Vahl, Symb. Bot. 2: 87 (1791)

$\equiv$ P. calcitrapifolium (Vahl) DC. in Lam. \& DC., Fl. Franç. ed. 3, 5: 455 (1815)

$\equiv$ S. laciniata var. calcitrapifolia (Vahl) Moris, Fl. Sardoa 2: 497 (1840-43)

$\equiv$ S. laciniata subsp. calcitrapifolia (Vahl) Maire in Bull. Soc. Hist. Nat. Afrique N. 22: 302 (1931)

= P. laciniatum var. latifolium Gren. \& Godr., Fl. France 2: 309 (1851)

$\equiv$ S. laciniata var. latifolia (Gren. \& Godr.)

Rouy in Rouy \& Foucaud, Fl. France 10: 17 (1908)]

\section{BIBLIOGRAFÍA}

DÍAZ DE LA GUARDIA, C. \& G. BLANCA-1987Revisión del género Scorzonera L. (Compositae, Lactuceae) en la Península Ibérica. Anales Jard. Bot. Madrid 43(2): 271-354.

MAVRODIEV, E.V., C.E. EDWARDS, D.C. ALBACH, M.A. GITZENDANNER, P.S. SOLTIS \& D.E. SOLTIS -2004- Phylogenetic relationships in subtribe Scorzonerinae
(Asteraceae: Cichorioideae: Cichorieae) based on ITS sequence data. Taxon 53(3): 699-712.

MAVRODIEV, E.V., M.A. GITZENDANNER, A.K CALAMINUS, R.M. BALDINI, P.S. SOLTIS \& D.E. SOLTIS -2012- Molecular phylogeny of Tragopogon L. (Asteraceae) based on seven nuclear loci (Adh, GapC, LFY, AP3, PI, ITS, and ETS). Webbia 67(2): 111-137.

OWEN, W.M., G. D'AMATO, R.I. DE DOMINICIS, P. SALIMBENI \& G.F. TUCCI -2006- A cytological and molecular study of the genera Scorzonera L. and Podospermum (L.) DC. (Asteraceae). Caryologia 59(2): 153-163.
Dirección de los autores. Departamento de Botánica. Facultad de Ciencias. Universidad de Granada. 18001 Granada. *Autor para correspondencia: cdiaz@ugr.es. 